\title{
ON A MARKOV ANALOGUE OF CONTINUOUS-TIME Q-PROCESSES
}

UDC 519.21

\author{
AZAM A. IMOMOV \\ Dedicated to the fond memory of Professor I. S. Badalbaev
}

\begin{abstract}
The so-called Markov continuous-time $Q$-processes are considered in the paper as a generalization of $Q$-processes. The asymptotic behavior of transition probabilities is studied for Markov $Q$-processes.
\end{abstract}

\section{INTRODUCTION}

Let $Z(t), t \geq 0$, represent the number of particles in a population at time $t$. Assume that $Z(t)$ is a homogeneous Markov branching process with the initial state $Z(0)=1$. Let

$$
P_{i j}(t):=\mathrm{P}\{Z(t+\tau)=j \mid Z(\tau)=i\}
$$

be the transition probabilities of the Markov branching process $Z$, where $t, \tau \geq 0$ and $i, j \in \mathbf{N}_{0}=\{0\} \cup\{\mathbf{N}=1,2, \ldots\}$. According to the fundamental property of branching processes, the evolution of states of a Markov branching process can be described in terms of the transition probabilities $P_{1 j}(t)$. In turn, the latter probability can be expressed in terms of local densities $\left\{a_{j}, j \in \mathbf{N}_{0}\right\}$ via the following relation:

$$
P_{1 j}(\Delta)=\delta_{1 j}+a_{j} \Delta+o(\Delta), \quad \Delta \rightarrow 0,
$$

where $\delta_{1 j}$ is the Kronecker symbol. The densities of transition probabilities are such that $a_{j} \geq 0$ for $j \in \mathbf{N}_{0} \backslash\{1\}$ and $0<a_{0}<-a_{1}$. Moreover, $\sum_{j \in \mathbf{N}_{0}} a_{j}=0$.

The role of such a sequence $\left\{a_{j}\right\}$ is explained by the following probabilistic model. Let the particles be of the same type and let each of them transform with probability $a_{k} \Delta+o(\Delta)$ into $k \in \mathbf{N}_{0} \backslash\{1\}$ particles during the time interval $(t, t+\Delta)$, while the number of particles does not change with probability $a_{1} \Delta+o(\Delta)$ during the same interval.

Like other models of branching processes, the main property for Markov branching processes is the law of transformation of particles into offspring governed by a control parameter $a:=\sum_{j \in \mathbf{N}_{0}} j a_{j}$. If $a=0$, then a Markov branching process is called critical, while if $a<0$ or $a>0$ it is called subcritical or supercritical, respectively.

In what follows we use the moment generating functions

$$
\Phi(t ; x):=\sum_{j \in \mathbf{N}_{0}} P_{1 j}(t) x^{j} \quad \text { and } \quad f(x):=\sum_{j \in \mathbf{N}_{0}} a_{j} x^{j}, \quad|x|<1 .
$$

2010 Mathematics Subject Classification. Primary 60J80.

Key words and phrases. Markov Q-processes, transition probability, stationary measures. 
The relationships between these moment generating functions can be written in the form of differential equations as follows:

$$
\frac{\partial \Phi(t ; x)}{\partial t}=f(\Phi(t ; x))
$$

and

$$
\frac{\partial \Phi(t ; x)}{\partial t}=f(x) \frac{\partial \Phi(t ; x)}{\partial x}
$$

with the initial condition $\Phi(0 ; x)=x$. Moreover, the moment generating function $\Phi(t ; x)$ satisfies the functional equation

$$
\Phi(t+\tau ; x)=\Phi(t ; \Phi(\tau ; x))
$$

for all $t, \tau \geq 0$ and with the same initial condition (see [8]).

It is known that the extinction probability $q$ of a Markov branching process equals the minimal nonnegative root of the equation $f(x)=0$. Moreover the extinction probability equals 1 if $a \leq 0$ and is less than 1 if $a>0$. Further, $1-\Phi(t ; x)=O(1 / t)$ in the critical case and $q-\Phi(t ; x)=O\left(\beta^{t}\right)$ in the noncritical case for all $|x|<1$ as $t \rightarrow \infty$, where $0<\beta:=\exp \left\{f^{\prime}(q)\right\}<1$. In particular, $\mathrm{P}\{Z(t)>0\}=1-\Phi(t ; 0) \rightarrow 0$ if $a \leq 0$. In other words, the distribution of $Z(t)$ converges to a degenerate distribution at zero if $a \leq 0$. This explains why almost all limit properties of Markov branching processes are studied under the condition that $Z(t)>0$. The same concerns the Galton-Watson process with discrete time (see [8]).

On the other hand, the results of the papers [4, [1, pp. 56-60], [5]-[6], and [2] show that if the trajectory of a process does not vanish asymptotically, then other limit theorems, different from the classical ones, may also hold. Note that the Galton-Watson processes whose trajectories do not vanish asymptotically are called $Q$-processes in the monograph [1].

In this paper, we consider the processes that are a generalization of $Q$-processes to the case of continuous time. By analogy with the case of discrete time, we call them the Markov $Q$-processes. A discussion concerning Markov $Q$-processes can be found in the author's paper [3].

\section{DeFinition AND PRELIMINARY REMARKS}

The definition of Markov $Q$-processes is similar to that of $Q$-processes. Assume that a trajectory of a Markov branching process does not vanish asymptotically. Define the stochastic matrix $\left\{Q_{i j}(t), i, j \in \mathbf{N}_{0}\right\}$ by passing to the limit as follows:

$$
Q_{i j}(t)=\lim _{r \rightarrow \infty} \mathrm{P}\{Z(t+\tau)=j \mid Z(\tau)=i, Z(t+\tau+r)>0\}
$$

for $t, \tau \geq 0$. Then

$$
Q_{i j}(t)=\frac{j q^{j-i}}{i \beta^{t}} P_{i j}(t)
$$

Consider a family of random variables $\{W(t), t \geq 0\}$ with transition probabilities $\mathrm{P}\{W(t+\tau)=j \mid W(\tau)=i\}=Q_{i j}(t)$. It is clear that the random variable $W(t)$ represents the number of particles in the population at a moment $t \geq 0$ in the Markov $Q$-process with transition probabilities $Q_{i j}(t)$.

To study the evolution of a Markov $Q$-process it is sufficient to know the probabilities $Q_{1 j}(t)$, since the definition involves the transition probabilities $P_{i j}(t)$ only. According 
to (1), the probabilities $Q_{1 j}(t)$ admit the following representation:

$$
\begin{gathered}
Q_{11}(\Delta)=1+q_{1} \Delta+o(\Delta), \\
Q_{1 j}(\Delta)=q_{j} \Delta+o(\Delta), \quad j \neq 1,
\end{gathered}
$$

as $\Delta \rightarrow 0$. The densities of the transition probabilities are such that

$$
q_{0}=0, \quad q_{1}=a_{1}-f^{\prime}(q)<0, \quad q_{k}=k q^{k-1} a_{k} \geq 0, \quad k \in \mathbf{N} \backslash\{1\} .
$$

Consider the moment generating functions

$$
G_{i}(t ; x):=\sum_{j \in \mathbf{N}} Q_{i j}(t) x^{j} \quad \text { and } \quad g(x):=\sum_{k \in \mathbf{N}} q_{k} x^{k} .
$$

It is easy to see that $g(x)=x\left[f^{\prime}(q x)-f^{\prime}(q)\right]$ and that this moment generating function is infinitesimal. Thus Markov $Q$-processes can uniquely be defined in terms of the moment generating function $g(x)$. In what follows we assume that the first moment $b:=\sum_{k \in \mathbf{N}} k q_{k}=g^{\prime}(1)$ is finite.

In view of (5), we get

$$
G_{i}(t ; x)=\frac{q x}{i \beta^{t}}\left[\frac{\partial}{\partial s}\left(\frac{\Phi(t ; s)}{q}\right)^{i}\right]_{s=q x}
$$

for all $t>0$. Equality (8) can be rewritten as follows:

$$
G_{i}(t ; x)=\left[\frac{\Phi(t ; q x)}{q}\right]^{i-1} G(t ; x)
$$

for all $i \in \mathbf{N}$. The moment generating function here is such that

$$
G(t ; x):=G_{1}(t ; x)=\mathrm{E}\left[x^{W(t)} \mid W(0)=1\right] .
$$

Since $q-\Phi(t ; x) \rightarrow 0$ as $t \rightarrow \infty$ for all $|x|<1$, it is sufficient to study the behavior of the moment generating function $G(t ; x)$ as $t \rightarrow \infty$ (see equality (9)). This means that the distribution approaches $Q_{1 j}(t)$ as the number of a generation increases.

It is shown in [3] that

$$
\frac{\partial \Phi(t ; q x)}{\partial x}=\exp \left\{\int_{0}^{t} f^{\prime}(\Phi(\tau ; q x)) d \tau\right\} .
$$

Substituting equality (10) in (8), we get

$$
G(t ; x)=x \exp \left\{\int_{0}^{t}\left[f^{\prime}(\Phi(\tau ; q x))-f^{\prime}(q)\right] d \tau\right\}
$$

for $i=1$.

The moment generating function $F(t ; x)$ of a Markov branching process with homogeneous immigration for which the moment generating function of the transformation density is $f(x)$ and the moment generating function of the intensity of immigrating particles is $b(x)$ admits the following representation:

$$
F(t ; x)=\exp \left\{\int_{0}^{t} b(\Phi(\tau ; q x)) d \tau\right\}
$$

(see [8]).

Put $b(x)=f^{\prime}(q x)-f^{\prime}(q)=\sum_{k \in \mathbf{N}} b_{k} x^{k}$. Since $b(x)=g(x) / x, b(x)$ is an infinitesimal moment generating function. Now equality (11) can be rewritten as follows:

$$
G(t ; x)=x \exp \left\{\int_{0}^{t} b\left(\frac{\Phi(\tau ; q x)}{q}\right) d \tau\right\} .
$$


Taking into account representation (12) and equality (13), the Markov $Q$-process can be described as a branching process with continuous time described as follows. At the beginning, there exists a unique particle. The evolution of the process starts due to the flow of immigrating particles governed by the moment generating function

$$
b(x)=f^{\prime}(q x)-f^{\prime}(q) .
$$

The particles arriving with the immigrating flow produce an offspring according to the moment generating function $f(q x)$. The initial particle does not disappear and does not produce an offspring. This particle lives during the infinite evolution of the process.

The above description makes clear that the studies of the asymptotic properties of a Markov $Q$-process reduce to those for a Markov branching process with homogeneous immigration.

The expectation $\mathrm{E} W(t)$ and variance $\operatorname{Var} W(t)$ can be evaluated by differentiating equality (13), namely

$$
\begin{aligned}
& \mathrm{E} W(t)= \begin{cases}b t+1, & a=0, \\
1+\gamma\left(1-\beta^{t}\right), & a \neq 0,\end{cases} \\
& \operatorname{Var} W(t)= \begin{cases}b t, & a=0, \\
\gamma\left(1-\beta^{t}\right), & a \neq 0,\end{cases}
\end{aligned}
$$

where $b=g^{\prime}(1)=f^{\prime \prime}(1)$ if $a=0$ and $\gamma:=q f^{\prime \prime}(q) /\left|f^{\prime}(q)\right|$ if $a \neq 0$. Equalities (14) and (15) imply that the parameter $a=f^{\prime}(1)$ is the principal characteristic of the evolution of a Markov $Q$-process.

Finally, using the functional equation (4) and the form of the moment generating function given in (9) the main functional equation can be written as follows:

$$
G_{i}(t+\tau ; x)=\frac{G(\tau ; x)}{G\left(0 ; \frac{\Phi(\tau ; q x)}{q}\right)} G_{i}\left(t ; \frac{\Phi(\tau ; q x)}{q}\right),
$$

where $G(0 ; x)=\lim _{t \rightarrow 0} G(t ; x)=x$.

\section{The stationarity of $W(t)$}

In this section, we study the limit behavior of the moment generating function $G_{i}(t ; x)$. As noticed above, it is sufficient to consider the moment generating function $G(t ; x)$. Putting $R(t ; x):=q-\Phi(t ; x)$ we derive from equality (8) that

$$
G(t ; x)=-\frac{x}{\beta^{t}} \frac{\partial R(t ; q x)}{\partial x}
$$

3.1. Case of $a \leq 0$. We have $q=1$ and $R(t ; x) \rightarrow 0$ as $t \rightarrow \infty$. Further, applying the results obtained in the paper [3] for the asymptotic representation of the function $\partial R(t ; x) / \partial x$ we prove the following auxiliary result.

Lemma 1. The moment generating function $G(t ; x)$ admits the following asymptotic representations:

1) if $a<0$, then

$$
G(t ; x)=e^{|a| t} \frac{|a| x}{f(x)} R(t ; x)(1+o(1)), \quad t \rightarrow \infty ;
$$

2) if $a=0$, then

$$
G(t ; x)=\frac{b x}{2 f(x)} R^{2}(t ; x)(1+o(1)), \quad t \rightarrow \infty .
$$


The value of the function $G(t ; x) / x$ at the point $x=0$ equals the probability $Q_{11}(t)$ of return to the initial state $\{W(0)=1\}$ over the time $t$. Rewriting the moment generating function $G(t ; x)$ in the form

$$
x \sum_{j \in \mathbf{N}} Q_{1 j}(t) x^{j-1}
$$

and considering (18) and (19) at the point $x=0$ we obtain the following local limit theorems by using the representation of $R(t ; x)$ (see [8]).

Theorem 1. Let $a<0$. Then

$$
Q_{11}(t)=\frac{|a| K}{a_{0}}(1+o(1)), \quad t \rightarrow \infty,
$$

where $K$ is the Sevast'yanov constant defined by

$$
\ln K=-\int_{0}^{1} \frac{a u+f(1-u)}{u f(1-u)} d u .
$$

We use the symbol $K$ for the Sevast'yanov constant throughout the paper.

Theorem 2. Let $a=0$. Then

$$
t^{2} Q_{11}(t)=\frac{2}{b a_{0}}(1+o(1)), \quad t \rightarrow \infty .
$$

The following two results follow from [3] and Lemma 1.

Theorem 3. Let $a<0$. Then

$$
G_{i}(t ; x)=\pi(x)(1+o(1)), \quad t \rightarrow \infty,
$$

where

$$
\pi(x)=K|a| \frac{x}{f(x)} \exp \left\{a \int_{0}^{x} \frac{d u}{f(u)}\right\} .
$$

The moment generating function $\pi(x)$ admits a representation in the form of a power series

$$
\pi(x)=\sum_{k \in \mathbf{N}} \pi_{k} x^{k}
$$

with the nonnegative coefficients $\left\{\pi_{n}, n \in \mathbf{N}\right\}$ forming the stationary distribution for the Markov $Q$-process. The transition probabilities $\left\{Q_{i j}(t), n \in \mathbf{N}\right\}$ admit the following asymptotic representation:

$$
Q_{i j}(t)=\pi_{j}(1+o(1)), \quad t \rightarrow \infty .
$$

Theorem 4. Let $a=0$ and let $\left\{Q_{i j}(t), n \in \mathbf{N}\right\}$ be the transition probabilities of a Markov $Q$-process. Then

$$
t^{2} G_{i}(t ; x)=\mu(x)(1+o(1)), \quad t \rightarrow \infty,
$$

where the limit moment generating function $\mu(x)=\sum_{j \in \mathbf{N}} \mu_{j} x^{j}$ is given by

$$
\mu(x)=\frac{2 x}{b f(x)} .
$$

The nonnegative coefficients $\left\{\mu_{j}, j \in \mathbf{N}\right\}$ are such that

$$
\mu_{i}=\sum_{j \in \mathbf{N}} \mu_{j} Q_{j i}(t)
$$

The transition probabilities of the Markov Q-process admit the following asymptotic representation:

$$
t^{2} Q_{i j}(t)=\mu_{j}(1+o(1)), \quad t \rightarrow \infty
$$


The following result follows from Theorem 4 .

Theorem 5. Let $a=0$. Assume that $\left\{\mu_{j}, j \in \mathbf{N}\right\}$ is the stationary measure of a Markov $Q$-process and that the first moment is finite, $b:=g^{\prime}(1)$. Then

$$
\lim _{n \rightarrow \infty} \frac{1}{n^{2}}\left[\mu_{1}+\mu_{2}+\cdots+\mu_{n}\right]=\frac{2}{b^{2}} .
$$

Relation (20) implies that $\sum_{j \in \mathbf{N}} \mu_{j}=\infty$. Therefore, there exists a unique stationary measure for a Markov $Q$-process if $a=0$.

3.2. Case of $a>0$. Now we consider the case of $a>0$, where the extinction probability of a Markov branching process is less than unity, that is, $q<1$. It is shown in [1, p. 115] that

$$
R(t ; x)=\beta^{t} A(x)(1+o(1)), \quad t \rightarrow \infty .
$$

The limit function $A(x)$ is a unique solution of the equation

$$
A(\Phi(t ; x))=\beta^{t} A(x)
$$

with $A(q)=0$ and $A^{\prime}(q)=-1$. Our aim is to write the function $A(x)$ explicitly.

We rewrite equation (2) as follows:

$$
R(t ; x)=(q-x) \beta^{t} \exp \left\{\int_{x}^{\Phi(t ; x)}\left[\frac{1}{s-q}-\frac{f^{\prime}(q)}{f(s)}\right] d s\right\} .
$$

Comparing (21) and (22), we conclude that

$$
A(x)=(q-x) \exp \left\{\int_{x}^{q}\left[\frac{1}{s-q}-\frac{f^{\prime}(q)}{f(s)}\right] d s\right\} .
$$

Putting $x=0$ in (23), we easily obtain that

$$
A:=A(0)=q \exp \left\{\int_{0}^{q}\left[\frac{1}{s-q}-\frac{f^{\prime}(q)}{f(s)}\right] d s\right\}<\infty .
$$

Equations (2) and (3) together with Taylor's formula imply that

$$
\frac{\partial R(t ; x)}{\partial x}=\frac{f^{\prime}(q)}{f(x)} R(t ; x)(1+o(1)), \quad t \rightarrow \infty .
$$

Combining (17), (21), and (25), we prove the following result.

Lemma 2. If $a>0$, then

$$
G(t ; x)=-x \frac{f^{\prime}(q)}{f(q x)} A(q x)(1+o(1)), \quad t \rightarrow \infty,
$$

where the function $A(x)$ is defined by (23).

Using Lemma 2 and equality (23), the limit value $U(x)=\lim _{n \rightarrow \infty} G_{i}(t ; x)$ is represented as follows:

$$
U(x)=\frac{q\left|f^{\prime}(q)\right|}{f(q x)} x(1-x) \exp \left\{\int_{q x}^{q}\left[\frac{1}{s-q}-\frac{f^{\prime}(q)}{f(s)}\right] d s\right\} .
$$

Considering $x=0$ we immediately derive the following local limit theorem from Lemma 2 and equality (26).

Theorem 6. Let $a>0$. Then

$$
Q_{11}(t)=\frac{\left|f^{\prime}(q)\right| A}{a_{0}}(1+o(1)), \quad t \rightarrow \infty,
$$

where the positive constant $A$ is defined by equality (24). 
As noticed above, the limit properties of a Markov $Q$-process can be studied via the corresponding Markov branching process with homogeneous immigration. Using this approach and a known result in the theory of Markov branching processes with homogeneous immigration one can obtain another (equivalent) representation for the limit moment generating function $U(x)$. The following result is proved in [7, Theorem 5].

Theorem A ([7]). Assume that $a>0$. Let a Markov branching process with homogeneous immigration be defined via the moment generating function (12). Then

$$
F(t ; x)=e^{b(q) t} C(x)(1+o(1)), \quad t \rightarrow \infty,
$$

for $|x| \leq q$, where

$$
C(x)=\exp \left\{\int_{x}^{q} \frac{b(s)-b(q)}{f(s)} d s\right\} .
$$

Following the lines of the proof of Theorem A and taking into account relations (9) and (13) we get the following result.

Theorem 7. Let $a>0$. Then

$$
G_{i}(t ; x)=U(x)(1+o(1)), \quad t \rightarrow \infty,
$$

where

$$
U(x)=x \exp \left\{\int_{q x}^{q} \frac{f^{\prime}(s)-f^{\prime}(q)}{f(s)} d s\right\} .
$$

The limit moment generating function $U(x)$ admits the expansion

$$
U(x)=\sum_{k \in \mathbf{N}} u_{k} x^{k}
$$

Furthermore, the transition probabilities $\left\{Q_{i j}(t), n \in \mathbf{N}\right\}$ admit the asymptotic representation

$$
Q_{i j}(t)=u_{j}(1+o(1)), \quad t \rightarrow \infty .
$$

A further reasoning leads to the conclusion that the limit moment generating function $U(x)$ given by (26) and the one obtained in Theorem 7 are the same.

Remark 1. The limit moment generating function $U(x)$ generates the stationary distribution for the Markov $Q$-process.

Indeed, the nonnegative coefficients $\left\{u_{n}, n \in \mathbf{N}\right\}$ satisfy the equation

$$
u_{i}=\sum_{j \in \mathbf{N}} u_{j} Q_{j i}(t)
$$

by the main functional equation (16). Moreover, $U(1)=\sum_{k \in \mathbf{N}} u_{k}=1$, since the limit

$$
\lim _{s \rightarrow q} \frac{f^{\prime}(s)-f^{\prime}(q)}{f(s)}=-\frac{\gamma}{q}
$$

is finite.

Remark 2. Equalities (14) and (15) allow one to conclude that $W(t)$ converges as $t \rightarrow \infty$ in the mean square sense and with probability one to a random variable $W$ such that

$$
\mathrm{E} W=1+\gamma \quad \text { and } \quad \operatorname{Var} W=\gamma
$$




\section{Limit THEOREM FOR THE CASE OF $a=0$}

We prove an analogue of a known Yaglom theorem on the limit law for the random variable $W(t) / \mathrm{E} W(t)$ in the case of $a=0$. The proof is similar to the discrete case (see 1, p. 59]). The result below is Theorem 7 of [3] stated accordingly to the case considered in this paper.

Theorem 8. Let $a=0$. Then

$$
\mathrm{P}\left\{\frac{W(t)}{\mathrm{E} W(t)} \leq x\right\} \rightarrow 1-e^{-2 x}-2 x e^{-2 x}, \quad t \rightarrow \infty,
$$

for all $x>0$.

\section{BIBLIOGRAPHY}

1. K. B. Athreya and P. E. Ney, Branching Processes, Springer, New York, 1972. MR0373040 $(51: 9242)$

2. A. A. Imomov, On a condition of the non-extinction for branching processes, Uzbek Matem. Zhurnal (2001), no. 2, 46-51. (Russian) MR.1943639 (2004c:60239)

3. A. A. Imomov, A differential analog of the main lemma of the theory of Markov branching processes and its applications, Ukr. Math. J. 57 (2005), no. 2, 307-315. MR2189330(2006i:60120)

4. J. Lamperti and P. E. Ney, Conditioned branching processes and their limiting diffusions, Theory Probab. Appl. 13 (1968), 128-139. MR0228073 (37:3657)

5. A. G. Pakes, Some limit theorems for the total progeny of a branching process, Adv. Appl. Probab. 3 (1971), no. 1, 176-192. MR 0283892 (44:1122)

6. A. G. Pakes, Some new limit theorems for the critical branching process allowing immigration, Stoch. Process. Appl. 3 (1975), 175-185. MR0397912 (53:1767)

7. A. G. Pakes, On Markov branching processes with immigration, Sankhyā: Indian J. Statist. 37(A) (1975), no. 1, 129-138. MR0433622 (55:6595)

8. B. A. Sevast'yanov, Branching Processes, Nauka, Moscow, 1971; German transl., Verzweigungsprozesse, Akademie-Verlag, Berlin, 1974. MR0345229 (49:9968)

Department of Probability Theory and Mathematical Statistics, Institute for Mathematics and Information Technologies, Academy of Sciences of Uzbek Republic, Do'rmon Yo'li Street 29, Tashkent 100125, UzBekistan

Current address: Department of Mathematical Analysis and Algebra, Karshi State University, Kuchabag Street 17, Karshi 180103, Uzbekistan

E-mail address: imomov_azam@mail.ru

Received 9/NOV/2009

Originally published in Russian

Translated by N. SEMENOV 